\title{
Keynote Talk Summary: Algorithmic Aspects of Sensor Networks
}

\author{
Paul Spirakis \\ Research Academic Computer Technology Institute, \\ University of Patras, Greece \\ spirakis@cti.gr
}

In this talk, we discuss some abstract models of sensor networks and also some basic algorithmic problems. We first go through the basic architecture and communication capabilities of a single, ultra small sized, sensor. We distinguish broadcast capability (at a certain radius $\mathrm{r}$ around the sensor) and directional broadcasting at an angle (which can be reduced to almost zero in case of optical communication). Based on such features, we first go through the (well known) model of Random Geometric Graphs and its threshold properties for connectivity, average degree, chromatic number etc. Then, we define the model of Random Sector Graphs, in order to capture unidirectional sending at an angle. We provide thresholds for connectivity, clique and chromatic number there also and point out its differences from the Random Geometric Graphs model. We also discuss other models related to ways of configuring sensor nets, such as "for each point (sensor) connect to its k nearest neighbours" or "for each sensor, connect to $\mathrm{k}$ points within its communication range $\mathrm{r}$ ". We also refer to the Combinatorial model of Random Intersection Graphs (which abstracts the geometry out). It is interesting to see that some properties (e.g., sensors density necessary for connectivity) are "invariant" in all these models. We then go through some fundamental algorithmic issues and their existing solutions. We first discuss the problem of finding efficient local protocols aiming in propagating a local event E to a sink. We discuss several efficiency measures like hop count, energy spent, short paths. Randomised local protocols are then sketched, useful for robust and energy efficient local event reporting in case of a sensor net with failures at some nodes (or with energy depleted nodes). In this framework we indicate some energy-time tradeoffs for propagation of local information and notice their similarity with tradeoffs between area and time in VLSI circuits. The problem of propagation of local information in areas with obstacles (lakes) is also discussed. We then examine efficient routing policies and especially refer to greedy techniques in the case of existence of virtual coordinates. The topology control problem is the next topic. We present several local protocols for configuring the net and establishing global properties like connectivity or short paths. Finally, we discuss problems related to scenarios where a sensor net is "embedded" into a larger fixed-connectivity network. There, we provide the abstraction of a "sensors cloud" and discuss the possible enhancement of properties like maximum flow or connectivity or diameter of the resulting hybrid network. Our talk aims in showing the emergence of a new algorithmic subfield, useful to the pragmatic considerations in the actual design and control of such networks.

The original version of this chapter was revised: The copyright line was incorrect. This has been corrected. The Erratum to this chapter is available at DOI: 10.1007/978-3-540-32993-0_29

I. Stavrakakis and M. Smirnov (Eds.): WAC 2005, LNCS 3854, p. 277, 2006.

(c) Springer-Verlag Berlin Heidelberg 2006 